\title{
Strates
}

STRATES Matériaux pour la recherche en sciences sociales

$11 \mid 2004$

Jeune recherche, la vitalité d'un laboratoire

\section{Une ville et ses urbanistes : Beyrouth en reconstruction}

\section{Eric VERDEIL}

\section{(2) OpenEdition \\ 12 Journals}

Édition électronique

URL : http://journals.openedition.org/strates/452

DOI : $10.4000 /$ strates. 452

ISSN : $1777-5442$

Éditeur

Laboratoire Ladyss

Édition imprimée

Date de publication : 1 janvier 2004

ISSN : 0768-8067

Référence électronique

Eric VERDEIL, "Une ville et ses urbanistes: Beyrouth en reconstruction », Strates [En ligne], 11 | 2004 , mis en ligne le 14 janvier 2005, consulté le 08 septembre 2020. URL : http://journals.openedition.org/ strates/452 ; DOI : https://doi.org/10.4000/strates.452

Ce document a été généré automatiquement le 8 septembre 2020

Tous droits réservés 


\title{
Une ville et ses urbanistes : Beyrouth en reconstruction
}

\author{
Eric VERDEIL
}

1 Thèse de géographie soutenue à l'université de Paris I le 4 décembre 2002, sous la direction de Pierre Merlin, professeur des universités, UMR Ladyss/Cnrs (654 p.)

2 Ce travail se situe dans le champ de l'histoire sociale

3 de l'urbanisme. Il se propose de saisir la généalogie des pratiques de l'aménagement urbain à la confluence entre la construction de systèmes normatifs et les déterminations économiques et politiques. La reconstruction après la guerre civile au Liban constitue à cet égard un observatoire privilégié : les reconstructions urbaines sont des moments d'expérimentation et de mise en œuvre des techniques d'urbanisme à une vaste échelle, dans un contexte de forte pression sociale et politique. Cela permet de s'interroger sur la place et la signification de l'urbanisme comme instrument politique dans les périodes de sortie de guerre. Néanmoins, la singularité d'un tel moment ne doit pas conduire à postuler son caractère exceptionnel au regard de ce que serait l'urbanisme ordinaire. Pour cela, la destinée particulière - et tragique -de la capitale du Liban doit être restituée dans un cadre de référence plus large, celui des politiques urbaines dans les pays arabes indépendants, sinon même plus largement, dans les pays émancipés des tutelles coloniales. L'aménagement urbain résulte pour une part des impératifs de la construction étatique nationale. La mise en pratique des techniques élaborées durant le moment colonial et adaptées ensuite par l'État indépendant en vue du développement économique s'effectue aussi dans le contexte d'une circulation complexe d'idées et de praticiens entre les pays occidentaux et les pays arabes, voire au-delà. La notion de cultures professionnelles de l'urbanisme s'avère très utile dans cette perspective. L'hypothèse principale de la recherche est que les pratiques d'urbanisme résultent d'une combinaison de temporalités de la société et de l'économie, de la politique et des cultures professionnelles.

4 La recherche s'est fondée sur des sources variées, situées tant au Liban qu'en France. À la documentation officielle libanaise, consultée auprès de diverses administrations en 
charge de l'urbanisme, se sont ajoutées l'étude des archives de bureaux d'études privés ayant exercé au Liban et une série d'entretiens avec des techniciens libanais.

5 La première partie de la recherche se concentre sur la période actuelle de la reconstruction et sur les déterminations spécifiques qu'elle entraîne pour les chantiers d'urbanisme de la période. Le premier chapitre est consacré à l'étude des caractères originaux de la reconstruction du centre-ville de Beyrouth, à partir de 1991. Adoptant le parti d'une quasi-table rase, l'opération apparaît en grande partie comme le projet d'un seul décideur, l'homme d'affaires devenu Premier ministre Rafiq Hariri, qui envisageait d'en faire le levier de la reconquête par le Liban de son rôle économique d'avant-guerre. En même temps, la réussite de ce projet et son caractère modèle étaient conçus comme les meilleurs arguments au service de sa carrière politique. Le projet tire son caractère de modèle, dans le Liban de la reconstruction, de la concentration exceptionnelle de pouvoirs par l'aménageur, qui le place en position d'assurer une grande cohérence aux travaux et d'en coordonner les étapes. Exceptionnel est également le montage juridique et financier, qui associe dans le capital de la société privée Solidere les anciens ayants droit et des investisseurs extérieurs. Le quasimonopole foncier qui en résulte permet d'éviter les risques ordinairement associés aux opérations d'urbanisme et les coûts d'une expropriation.

6 Le deuxième chapitre est consacré aux controverses qui ont entouré ce projet et aux évolutions qu'elles ont entraînées. Une partie des opposants initiaux - ceux qui avaient le plus grand poids politique et économique - ont été ralliés par diverses concessions et avantages visant à leur rendre acceptable l'expropriation jugée abusive. Au-delà des arguments financiers, le débat porta aussi sur les enjeux politiques, urbanistiques et sociaux du projet. Les critiques furent le fait d'une opposition intellectuelle qui comprenait notamment une partie de l'élite professionnelle des architectes et des urbanistes. Pour l'essentiel, ces contestations demeurèrent sans effet. Au contraire, le front des opposants se déchira au fur

et à mesure que le projet se concrétisait, dévoilant la diversité des intérêts sociaux et des références alternatives de l'opposition, ce qui implique de mieux les identifier.

Auparavant, afin de mettre en perspective ces enjeux et ces débats, le troisième chapitre analyse les destructions et les transformations sociales dues à la guerre dans une ville profondément divisée selon des lignes confessionnelles, et les projets de reconstruction proposés pour y remédier à l'échelle de l'agglomération et en divers lieux de la capitale. L'architecture administrative de la reconstruction traduit la diversité des objectifs politiques, économiques et sociaux assignés aux politiques de reconstruction durant la guerre. Dans certains cas, comme celui du réaménagement de l'ancienne ligne des combats, la divergence des visions s'oppose à l'émergence d'un projet de reconstruction. Dans d'autres cas, comme celui du transport urbain, les choix financiers et les représentations dominantes en faveur de l'automobile conduisent à minimiser les enjeux de la réunification de l'agglomération.

9 Deux raisons justifient le choix de consacrer la deuxième partie à une analyse historique de la condition de l'urbanisme au Liban : d'abord, les références régulières, dans les débats de la reconstruction, à la situation économique antérieure du Liban et de la capitale ainsi qu'aux anciens projets d'aménagement, en particulier ceux de la période chehabiste. Celle-ci représente en outre un exemple de convergence autour des pratiques d'aménagement spatial et constitue une sorte de miroir pour la période de sortie de guerre. 
10 Le quatrième chapitre s'attache aux politiques d'aménagement spatial dans le Liban de l'Indépendance. L'héritage des périodes ottomane et mandataire consistait essentiellement dans la réforme des outils juridiques et l'affirmation de la nécessité d'une modernisation urbaine. Le contexte politique de l'Indépendance, qui consacrait la force des milieux d'affaires hostiles à l'intervention de l'État dans l'économie, n'y était guère plus favorable. Toutefois, quelques tentatives furent faites durant la mandature Chamoun (1952-58), dont un effort d'aménagement régional des zones touchées par le tremblement de terre de 1956 et des travaux d'équipement urbain à Beyrouth. Le milieu technique local, quoique peu préparé aux tâches de l'aménagement urbain, s'y trouva progressivement impliqué et certains ingénieurs tentèrent d'en théoriser les principes.

11 Le cinquième chapitre, consacré aux projets du président Fouad Chehab (1958-64), représente un tournant majeur. Soucieux de promouvoir un développement équilibré du pays, le président encouragea des réformes institutionnelles profondes et entreprit un effort d'aménagement du territoire. Conseillé par l'Institut de recherche et de formation en vue du développement (IRFED) fondé par le père dominicain Louis-Joseph Lebret, Chehab soutint l'établissement d'un plan quinquennal et favorisa l'équipement des régions périphériques. Dans le domaine de l'urbanisme, il confia à l'urbaniste Michel Écochard le soin de proposer un plan d'urbanisme pour l'aménagement du Grand Beyrouth.

12 Le sixième chapitre s'attache au milieu professionnel impliqué dans l'urbanisme à l'époque de Chehab. Les projets des experts français furent généralement bien accueillis car ils répondaient à des préoccupations fortes des techniciens locaux. Toutefois, certaines réticences se manifestèrent parmi eux, notamment à l'égard de l'IRFED. Elles exprimaient à la fois le sentiment d'une concurrence entre experts locaux et étrangers et une méfiance face à certaines conceptions que véhiculaient ces derniers. Dans les années suivantes, le développement de la commande en urbanisme, sous l'impulsion de l'État, constitua un nouveau marché et une opportunité pour un milieu professionnel en croissance numérique rapide. Les « urbanistes », en fait architectes ou ingénieurs généralistes, diplômés après 1945, se recrutaient essentiellement parmi l'élite sociale de ces professions. L'examen des plans montre une double préoccupation : le souci d'une modernisation essentiellement technique, en vue d'un développement du pays par le secteur des services; et celui d'affirmer la place des techniciens, face aux politiques, dans la définition même des orientations du développement national.

13 Le chapitre sept entreprend un bilan des actions réformatrices et des réalisations spatiales de la période. L'examen du plan Ecochard pour Beyrouth et ses banlieues permet d'analyser l'interaction entre planificateurs, personnel politique et intérêts privés. Si ces derniers s'opposent fortement aux ambitions planificatrices, un changement graduel de perception est néanmoins perceptible : de nouvelles normes de gestion de la construction sont ainsi introduites. Henri Eddé, figure centrale du milieu, nommé ministre des Travaux publics en 1970 et à ce titre responsable de l'urbanisme, fit néanmoins l'expérience de la marge de manœuvre limitée laissée aux techniciens dans le contexte de la fin du chehabisme face aux intérêts privés. En revanche, la direction générale de l'urbanisme se dota progressivement de nouveaux outils. Le déclenchement de la guerre ne lui laissa pas l'occasion de mettre à l'épreuve ces nouvelles ambitions. 
14 Malgré le reflux des idées interventionnistes, un changement graduel des normes en matière d'urbanisme était intervenu. La troisième partie a pour objectif de cerner dans quelle mesure cet héritage influe sur les politiques urbaines volontaristes mis en place durant la guerre et la reconstruction.

15 Le chapitre huit fait le bilan des renouvellements de la guerre. Dès la paix manquée de 1977, la nécessité de repenser l'action en matière d'aménagement, face au défi des ruines et des bouleversements de la guerre, imposa une nouvelle organisation administrative. Le Conseil de développement et de la reconstruction en constituait le fleuron, et fut notamment mis à contribution par le président Amine Gemayel, aux commandes du pays lors d'une seconde tentative de reconstruction en 1982-83. Néanmoins, de nombreuses continuités inspiraient, en matière d'urbanisme, la définition des politiques urbaines, même si les nouveaux rapports de force imprimaient, directement ou indirectement, leur marque sur l'élaboration des projets et des plans d'aménagement, comme le montra le schéma directeur de la région métropolitaine

16 de Beyrouth entre 1983 et 1986. La guerre entraîna aussi des bouleversements sur la scène professionnelle, et favorisa la montée en puissance de nouvelles structures, à cheval sur les marchés libanais et l'étranger, où elles acquéraient un savoir-faire propre à traiter les défis de la reconstruction, comme le montrait l'ascension du bureau d'études Dar al-Handasah sur la scène urbanistique.

17 Les trois derniers chapitres illustrent, par l'étude des projets d'aménagement pour trois sites majeurs pour l'urbanisme beyrouthin, l'articulation entre déterminations de la guerre et de la reconstruction et idées anciennes dans l'élaboration des projets d'aménagement. $\mathrm{Au}$ centre-ville, dès les années soixante, des projets de réaménagement s'efforçaient de combiner volonté de modernisation économique et inscription dans l'espace, par l'activité planificatrice, de l'autorité étatique. Les dégâts de la guerre entraînèrent un approfondissement graduel des projets restés inachevés et l'exploration de solutions techniques et juridiques autour du thème de la société foncière. La désagrégation de l'État

18 et la montée de nouveaux acteurs, symbolisés par la figure du promoteur Rafiq Hariri, conduisirent à l'adoption de solutions radicales préfigurant les choix drastiques de la reconstruction de l'après-guerre.

19 La banlieue sud-ouest fit longtemps figure de laboratoire de l'urbanisme beyrouthin, au sens d'un lieu pour lequel était élaboré un modèle urbanistique et social de référence et étaient expérimentés des outils d'action nouveaux, notamment les remembrement et la société foncière ; et au sens d'un banc d'essai, en raison des difficultés rencontrées lors de leur mise en œuvre. La guerre et le développement des occupations irrégulières en consacraient l'échec. En même temps, s'exprimait la permanence d'un projet social et politique exclusiviste. Les rapports de force dans la reconstruction imposèrent un compromis où se mêlaient innovations et attitudes traditionnelles, sans que finalement les réalisations n'aboutissent.

20 Les remblais constituent un dernier thème d'étude, à travers l'exemple du littoral nord de l'agglomération. En un sens, ces projets sont une réponse aux restructurations de l'agglomération dans la guerre. Leur conception est également étroitement liée à la destinée politique des dirigeants successifs du pays, A. Gemayel puis R. Hariri. En outre, ces projets étaient liés à la volonté de divers groupes d'accaparer des ressources 
foncières qui apparaissaient comme l'une des principales richesses dans le Liban d'après-guerre. Toutefois, se surimposant à cette première lecture, plusieurs exemples montrent que les remblais furent conçus, dès avant la guerre, dans les milieux professionnels de l'urbanisme, comme un outil de développement devant pallier les lacunes des outils juridiques comme la société foncière, en permettant l'acquisition, au meilleur prix, de surfaces foncières mobilisables dans des projets d'aménagement conformes à certains idéaux professionnels et au développement économique du pays. Ces études de cas illustrent la combinaison de temporalités qui rend compte des formes prises par les politiques d'urbanisme de la reconstruction: temps long des cultures professionnelles, moyenne durée des changements économiques et sociaux dus à la guerre, temps court des enjeux politiques de la guerre ou de la reconstruction.

INDEX

Index géographique : Liban

Mots-clés : Ville 